\section{Detecting Earth-like planets}

SIR-Angel et al. ${ }^{1}$, while advocating the use of a $16 \mathrm{~m}$ diameter telescope optimized for infrared observations to search for Earth-like planets outside the Solar System, criticize my suggestion ${ }^{2}$ of using an interferometric system, operating at optical wavelengths, for the same purpose. Their criticism supposes that the sidelobe levels would be objectionably high because of the optical imperfections in the interferometry elements but here we point out that there is a fundamental error in their analysis.

Sidelobe suppression is an important issue for both concepts. The interferometer that I discussed would consist of a number of telescopes of diameter $D$, deployed in an array with spacings larger than $D$, but not so large that a planetary disk would be resolved. The elements would be pointed away from, but still close by, the star in searching for planets, but photons from the star would cause a noise background because of the finite sidelobe response. The star could be used as a phase reference to stabilize the interferometer, thus allowing coherent integration, and the diffraction sidelobes would be minimized by apodizing the aperture illumination of the individual elements (which were $1.5 \mathrm{~m}$ telescopes in the example given). Still, there would be noise sidelobes introduced by surface irregularities in the optics and it is control of these sidelobes that was being questioned by Angel et al.

To summarize the causes of noise sidelobes, the usual definition of gain will be used; relative gain (sometimes called directivity) is the gain normalized to unity on the optical axis (this is the inverse of the definition given by Angel et al.). The gain contribution from the noise sidelobes at an angle $(\theta)$ off the optical axis will be denoted by $G_{\mathrm{n}}(\theta)$. Intensity in the image plane will be the Fourier transform of the auto-correlation of the amplitude error in the aperture-plane phase front. The precise form of the sidelobes will depend upon the error distribution, but as a representative case let the errors have a random distribution, with an auto-correlation of the form

$<y^{2}(\tau)>=<\delta^{2}>1-\mathrm{e}^{-(2 \pi n)}$

for separation $\tau$ : $\iota$ is a correlation length and is the phase error. If the r.m.s. surface error is $\varepsilon$, then $\left\langle\delta^{2}\right\rangle=(4 \pi \varepsilon / \lambda)^{2}$. It then follows from the work of Ruze ${ }^{3}$ that, for a uniformly illuminated aperture of diameter $D$ with $\delta<<\lambda$, the relative gain of the noise sidelobes will have the distribution

$G_{\mathrm{n}}(\theta)>=8 \pi^{2}(\iota / D)^{2}(\varepsilon / \lambda)^{2} \mathrm{e}^{-(1 / 2 \pi \mid \theta / /)^{2}}$
Physically, the gain distribution will be a speckle pattern extending over an angle $\approx(\lambda / l)$ with individual speckles of size $\approx(\lambda / D)$.

In their remark that "The speckles will look like a cluster of barely resolved stars of 21 st magnitude, in which is hidden a 28th magnitude planet" Angel et al. misunderstand interferometry. The speckle pattern is derived from spatial frequencies less than $(\lambda / D)$, whilst the interferometer fringes come from spatial frequencies much larger than $(\lambda / D)$. Hence the interferometer fringe amplitude comes from the planet and not from the speckles, which only contribute the noise that was accounted for in the signal-to-noise calculation. Their assertion is, therefore, incorrect.

Angel et al., in estimating noise sidelobes from their equation (1) for the quantity they call the gain, illuminate the fundamental issue. Equation (2) shows that the distribution of errors must be considered. The total power scattered from the main beam depends only upon $\varepsilon$, and if, for a given $\varepsilon, l$ is small, the scattered power is distributed over a large angle, and the close-in sidelobe level will be low. That is why I chose $l=1 \mathrm{~cm}$ as the correlation length, giving a sidelobe suppression $1.6 \times 10^{3}$ greater than the example given by Angel et al.

On the other hand, if the only errors in the phase front were from large spatial frequencies, their objection would be well-taken. A beautifully polished surface, whose only errors in figure were on large scales, would be unsuitable for finding planets since all the noise sidelobes would be clustered close to the main beam. Thus, the example quoted by Angel et al., who used $l=40 \mathrm{~cm}$ as the correlation length in their calculation, is concentrating on the wrong regime. Note that, in my discussion, a mirror diameter of $1.5 \mathrm{~m}$ was chosen for the receiving elements, because of the extensive industrial experience in making high-quality mirrors in this size range. Industrial suppliers, in informal inquiries, have indicated that achieving this precision of the element surfaces is an economic, not a technical, question.

In summary, it is far too early to choose between the optical interferometer and the monolithic infrared telescope for a planetary search, as both the optical and infrared approaches need more extensive study. The challenges are well-defined and there are no fundamental physical laws that forbid their solution.

Department of Physics,

Massachusetts Institute of Technology, Cambridge, Massachusetts 02139, USA

1. Burke, B.F. Nature 322, $340-341$ (1986)

2. Angel, J.R.P., Cheng, A.Y.S. \& Woolf, N.J. Nature 322. $341-343$ (1986).

3. Ruze, J. Proc. Instit. elect. electron. Engng 54, 633-640 (1966).
ANGel et al. reply-Burke has defined a mirror surface by equation (1) with $l=1 \mathrm{~cm}$, showing that a relatively large r.m.s. error can be combined with very low sidelobes at $1 / 4$ arc second radius. But this bears little relationship to the best optical surfaces currently made. In practice, errors are not confined to small scales of 'orange peel' and 'dog biscuit'. Zonal figuring, in particular, leaves circular waves on the crucial scale of $40 \mathrm{~cm}$ $(\lambda / l=1 / 4$ arc $s)$. For optical detection of a planet at this radius with apodized $1.5 \mathrm{~m}$ mirrors, sidelobes of 10-7 peak intensity need surface accuracy of about $1 \AA$ r.m.s. on a scale of $40 \mathrm{~cm}$, regardless of finer imperfections. By comparison, figuring errors on this scale are about $50 \AA$ r.m.s. in the Hubble Space Telescope mirror. We would find the case for optical planetary detection more convincing if suggestions were given for dramatic breakthroughs in figuring, testing and support methods.

Does the use of an interferometer help, given an overwhelming need for high contrast rather than spatial resolution? For overcoming the photon noise of scattered light it offers at best no advantage over a single dish of the same area. The array gain cannot be better than the value of $D^{2} \theta^{2} / 8 \pi \delta^{2}$ given in our paper, where $D$ is the diameter of the single dish that collects the same light. The character of the background is the same as the specklefringe convolution familiar to observers with ground-based optical interferometers $^{i}$, except at an appropriately reduced intensity level. As in the case for the single $8 \mathrm{~m}$ dish analysed in our paper, by morphology there is no way to distinguish a planet from the much brighter scattered starlight fringes. Only if the interferometer baseline is $40 \mathrm{~m}$ or more will a solartype star at 4 pc be resolved and the scatterd light modulation be reduced. But an interferometer with so large a baseline, if arranged for good ultraviolet plane sampling, loses efficiency. Much of the planetary energy appears in parts of the diffraction pattern of low surface bright$n^{n} s^{2}$, and the signal to noise ratio becomes much less than optimum.

We conclude that neither the optical spectrum nor interferometry are suited to this problem. The infrared telescope with filled aperture that we described has improved contrast and reduced scattered light for a given figuring precision, and can be accurately aligned by correcting for errors sensed at optical wavelengths.

ROGER ANGEL ANDREW Cheng Neville Woolf

Steward Observatory,

The University of Arizona,

Tuscon, Arizona 85721, USA

1. Hege, E.K., Beckers, J.M. Strittmatter, P.A. \& McCarthy, D.W. Appl. Opt. 24, 2565 (1985)

2. Meinel, A.B., Meinel, M.P. \& Woolf, N.J. Appl. Opt. opt. Engng 10, 150 (1983). 